\title{
COMBINATION OF BILATERAL FILTER AND MATHEMATICAL MORPHOLOGY TO DETECT IMPACT CRATER ON MARS SURFACE
}

\author{
MIRIAM M. PEDROSA ${ }^{1}$, AYLTON PAGAMISSE ${ }^{2}$, ERIVALDO A. SILVA ${ }^{3}$.
}

\author{
1. Programa de Pós-graduação em Ciências Cartográficas, Universidade Estadual Paulista Julio de \\ Mesquita Filho \\ Rua Roberto Simonsen, 305 - Campus Universitário - 19060-900 - Presidente Prudente - SP \\ E-mail: miriammmphotmail.com
}
2. Depto. de Matemática e Computação, Universidade Estadual Paulista Julio de Mesquita Filho Rua Roberto Simonsen, 305 - Campus Universitário - 19060-900 - Presidente Prudente - SP
E-mail: aylton@fct.unesp.br

\author{
3. Depto. de Cartografia, Universidade Estadual Paulista Julio de Mesquita Filho \\ Rua Roberto Simonsen, 305 - Campus Universitário - 19060-900 - Presidente Prudente - SP \\ E-mail: silva.erivaldo@gmail.com
}

\begin{abstract}
Remotely sensed images have offered support to studies of lunar and planetary surfaces. Consequently, a large number of images have been acquired, and the amount of information increases in the same proportion. Therefore, there is a need for methodologies which perform the detection of the information contained in these images. Among the structures present in the lunar and planetary surfaces, the most notable are impact craters. The digital image processing assumes crucial importance when it comes to interpreting, detecting and extracting information in remotely sensed images. Thus, this paper presents a methodology for detection of impact craters on Mars, through two techniques, mathematical morphology and bilateral filter. This methodology was divided in three main stages: preprocessing, in which we apply the bilateral filter to smooth the image, in order to increase the difference between the targets of interest and irrelevant targets, for this paper. The second step is filtering phase, in which the goal is to exclude all the targets that aren't impact craters. Lastly, the segmentation phase was applied to separate craters that were close.
\end{abstract}

Keywords— Mathematical morphology, bilateral filter, digital image processing, Mars's impact crater.

\begin{abstract}
Resumo - As imagens de sensoriamento remoto oferecem suporte para estudos de superfícies planetárias e lunar. Consequentemente, um grande número de imagens tem sido adquirido, e a quantidade de informações nessas imagens crescem na mesma proporção. Assim, existe a necessidade de metodologias que façam a extração de informações contidas nessas imagens. Dentre as estruturas presentes na superfície lunar e planetárias, as crateras de impacto são as que mais se destacam. O processamento digital de imagens assume importância crucial quando se trata de interpretar, detectar e extrair informações em imagens de sensoriamento remoto. Este artigo apresenta uma metodologia para detecção de crateras de impacto na superfície de Marte, por meio de técnicas de morfologia matemática juntamente com o filtro bilateral. Esta metodologia foi dividida em três etapas principais: pré-processamento, no qual o filtro bilateral foi aplicado com objetivo de suavizar a imagem, para aumentar a diferença entre os alvos de interesse e alvos irrelevantes, para esse artigo. A segunda etapa é a fase de filtragem, na qual o objetivo é excluir todos os alvos que não se caracterizam como crateras de impacto. Por fim, a fase de segmentação foi aplicada, para separar crateras que estavam próximas.
\end{abstract}

Palavras-chave—Morfologia matemática, filtro bilateral, processamento digital de imagens, crateras de impacto de Marte.

\section{Introdução}

Crateras de impacto são estruturas formadas pela colisão de meteoritos com a superfície de um planeta. Elas são as que mais se destacam dentre as demais estruturas presentes nas superfícies dos planetas telúricos, exceto a Terra. O planeta Terra também sofreu bombardeios, principalmente durante o processo de formação do sistema solar, porém devido os processos de erosão e sedimentação, bem como os de atividade vulcânica e tectônica, grande parte das crateras de impacto foram apagadas (CRÓSTA, 2006).
A importância das crateras de impacto se justifica pela quantidade de informações que uma análise detalhada da quantidade e de sua morfologia pode trazer, como por exemplo, na ausência de medidas feitas in situ, contagem de crateras de impacto é a única técnica para o estabelecimento da cronologia relativa de diferentes superfícies planetárias (STEPINSKI, 2012).

As crateras de impacto na superfície marciana tem despertado o interesse de muitos pesquisadores. Agências de pesquisas espaciais têm enviado sondas com capacidade de aquisição de dados cada vez mais precisa, o que possibilita estudos que não eram possíveis com dados de resolução inferiores. Desta forma, sensores com alta capacidade de aquisição de 
informação tem gerado um banco com milhares de imagens, o que exige métodos automáticos para detecção de alvos de interesse.

Neste sentido, este trabalho se baseia na utilização de duas ferramentas do processamento digital de imagens, a morfologia matemática e o filtro bilateral, com vista à detecção de crateras de impacto na superfície do planeta Marte.

\section{Propósito}

O objetivo desse trabalho foi combinar duas ferramentas do processamento digital de imagens, filtro bilateral e morfologia matemática, para detectar crateras de impacto na superfície de Marte.

\section{Fundamentação teórica}

\subsection{Morfologia Matemática}

A morfologia matemática é uma teoria que foi desenvolvida por dois matemáticos francêses, George Matheron e Jean Serra, na École Nationale Supérieure des Mines de Paris, por volta de 1964.

A morfologia matemática é uma teoria que vem sendo amplamente utilizada na área de processamento digital de imagens e se destaca pelo fato de poder ser utilizada em uma série de finalidades, tais como, realce, filtragem, segmentação, esqueletização, dentre outras.

Essa teoria foi inicialmente criada para processamento em imagens binárias, com base na teoria dos conjuntos. Em seguida, utizando as relações de ordem, essa teoria foi estendida para imagens em níveis de cinza, a qual foi baseada na teoria dos reticulados.

Segundo Facon (1996), o princípio básico da morfologia matemática consiste em extrair informações relativas à geometria e à topologia de um conjunto desconhecido da imagem, por meio de um conjunto completamente conhecido, tanto seu tamanho como sua forma, esse conjunto é denominado elemento estruturante.

A morfologia matemática possui dois operadores que são fundamentais, a erosão e a dilatação, a partir dos quais todos os operadores podem ser derivados.

\subsubsection{Erosão}

De acordo com Soille (2003), a erosão de uma imagem $f$ por um elemento estruturante $\mathrm{B}$, é definida como sendo o valor mínimo da imagem, na janela definida pelo elemento estruturante, quando sua origem está em $x$.

$$
\left[\varepsilon_{B}(f)\right](x)=\min _{b \in B}(f(x+b)-B(b))
$$

\subsubsection{Dilatação}

De acordo com Soille (2003), a dilatação de uma imagem $f$ por um elemento estruturante $\mathrm{B}$, é definida como sendo o valor máximo da imagem, na janela definida pelo elemento estruturante, quando sua origem está em $x$.

$$
\left[\delta_{B}(f)\right](x)=\operatorname{ma}_{b \in B}(f(x+b)+B(b))
$$

\subsubsection{Gradiente}

O gradiente é uma operação que pode ser utilizada para detectar mudanças de tonalidades acentuadas, as quais normalmente caracterizam as bordas dos objetos.

A partir dos operadores básicos da MM Soille (2003) apresenta três combinações que são atualmente utilizadas na representação de bordas: diferença aritmética entre a dilatação e a erosão, diferença aritmética entre a dilatação e a imagem original e diferença aritmética entre a imagem original e a erosão.

O gradiente aplicado neste trabalho foi a diferença aritmética entre a dilatação e a erosão:

$$
\rho_{B}(f)=\delta_{B}(f)-\varepsilon_{B}(f)
$$

\subsubsection{Abertura}

A abertura de uma imagem $f$ por um elemento estruturante B é definida como a erosão de $f$ por B, seguida da dilatação de $f$ com o elemento estruturante transposto, $\widetilde{\boldsymbol{B}}$, (SOILLE, 2003):

$$
\gamma_{B}(f)=\delta_{\breve{B}}(f)\left[\varepsilon_{B}(f)\right]
$$

\subsubsection{Fechamento}

O fechamento de uma imagem $f$ por um elemento estruturante B é definida como a dilatação de $f$ por B, seguida da erosão de $f$ com o elemento estruturante transposto, $\breve{B}$, (SOILLE, 2003):

$$
\phi_{B}(f)=\varepsilon_{\breve{B}}(f)\left[\delta_{B}(f)\right]
$$

\subsubsection{Transformação Distancia}

A transformação distância atribui a cada pixel pertencente ao objeto, em uma imagem binária, a distância entre esse pixel e o pixel pertencente ao fundo da imagem, mais próximo.

A transformação distância é definida por Lotufo (2003) da seguinte maneira: considere A um conjunto e $x$ um ponto pertencente a $\mathrm{A}$, a $\mathrm{TD}(\mathrm{A})(x)$ é a distância a partir de $x$ para o complemento de A:

$$
T D(A)(x)=\min \left\{d(x, y), y \in A^{c}\right\}
$$




\subsubsection{Máximo Regional}

De acordo com Soille (2003), um máximo regional $m$ de uma imagem $f$ de elevação $t$ é um componente conectado, de pixels com valor $t$, cujos pixels da borda externa têm valores estritamente menores que $t$. Isto é alcançado através da subtração da reconstrução da imagem por dilatações a partir de $(f+1)$, da imagem original, como é mostrado na equação:

$$
R M A X=f-R_{f}^{\delta}(f-1)
$$

\subsubsection{Transformação watershed}

A transformação watershed associado ao conjunto dos mínimos regionais, $\boldsymbol{M}=\mathrm{U}_{\boldsymbol{i} \in \mathbb{R}} \boldsymbol{m}_{\boldsymbol{i}}$, de uma imagem $\boldsymbol{f}$, é o complemento da união de todas as bacias de retenção, $\boldsymbol{c}_{\boldsymbol{f}}\left(\boldsymbol{m}_{\boldsymbol{i}}\right)$, (PRÉTEUX, 2003):

$$
W L(f)=\left[\bigcup_{i \in R} C_{f}\left(m_{i}\right)\right]^{c}
$$

\subsection{Filtro Bilateral}

O filtro bilateral foi proposto originalmente por Tomasi e Manduchi em 1998, como uma ferramenta para remoção de ruídos. Esse filtro possui a característica de remover ruídos preservando as bordas dos objetos da imagem. O filtro bilateral é definido como (ZHANG, 2009):

$$
\tilde{I}(X)=\frac{1}{C} \sum_{y \in N(x)} e^{\frac{-\|y-x\|}{2 \sigma_{d}^{2}}} e^{\frac{-\|I(y)-I(x)\|^{2}}{2 \sigma_{r}^{2}}} I(y)
$$

Onde $\sigma_{d}$ e $\sigma_{r}$ são parâmetros que controlam o peso no domínio espacial e de intensidade, respectivamente, I(x) representa os pixels da imagem, $\mathrm{N}(\mathrm{x})$ é uma vizinhança espacial de $\mathrm{I}(\mathrm{x})$, e $\mathrm{C}$ é a constante de normalização representada pela seguinte expressão:

$$
C=\sum_{y \in N(x)} e^{\frac{-\|y-x\|}{2 \sigma_{d}^{2}}} e^{\frac{-\|I(y)-I(x)\|^{2}}{2 \sigma_{r}^{2}}}
$$

O filtro bilateral tem a característica principal de suavizar a imagem sem suavizar as bordas dos alvos. Além disso, ele também pode ser aplicado para a redução do efeito de blocking (ZHANG, 2009).

\section{Método}

Este trabalho compreende as seguintes etapas:

1. Pré-processamento: Nesta etapa vários filtros de suavização de imagens foram testados, tais como, filtros morfológicos, filtro da média, filtro mediano e o filtro bilateral. O objetivo nesta fase foi verificar qual a melhor técnica para a redução de ruídos.

2. Filtragem: Nesta fase foi feita uma binarização e em seguida foram aplicados operadores morfológicos com objetivo de eliminar alvos não circulares das imagens.

3. Segmentação: aplicou-se a transformação watershed, com objetivo de isolar alvos que se encontravam próximos e foram detectados como um único alvo.

\subsection{Pré-processamento}

Para o pré-processamento, vários filtros de redução de ruídos foram testados, com o objetivo de analisar qual realçaria mais as crateras de impacto em relação aos demais alvos presentes nas imagens. Os filtros testados foram os filtros morfológicos: volume máximo e mínimo, o filtro da média, o filtro mediano e o filtro bilateral. Os filtros que apresentaram melhores resultados foram os filtros morfológicos e o bilateral. O resultado da aplicação desses filtros é mostrado na Figura 1.

Os operadores morfológicos utilizados foram o volume mínimo e o volume máximo, com limiares 30 e 120 respectivamente. A finalidade do operador volume mínimo é remover bacias com volume menor do que o valor do limiar estipulado, utilizando para isso o critério de contraste. Analogamente, o operador volume máximo remove cúpulas com volume menor do que do limiar estipulado. O resultado da aplicação dos operadores morfológicos é mostrado na Figura 1 (a).

O filtro bilateral foi aplicado com os seguintes parâmetros: tamanho da janela 20, peso no domínio espacial 50 e peso no domínio de frequência 5. De acordo com Zhang (2009), não existe estudos mostrando as relações entre esses dois parâmetros, portanto, esses valores foram escolhidos empiricamente. O resultado da aplicação do filtro bilateral é mostrado na Figura 1 (b).

Como esses dois filtros apresentaram melhores resultados, foi desenvolvida uma rotina, e os resultados desses dois filtros foram testados para analisar qual auxiliaria melhor a tarefa de detecção de crateras de impacto.

Em seguida, aplicou-se o gradiente morfológico, o qual tem como objetivo aumentar as variações de níveis de cinza, visto que as bordas das crateras possui a característica de se destacar em relação a sua vizinhança, devido a alta variação de níveis de cinza.

Após as bordas das crateras terem sido destacadas do restante da imagem, as imagens foram binarizadas, o limiar utilizado foi 15 , esse limiar foi escolhido com base na análise do histograma da imagem. 


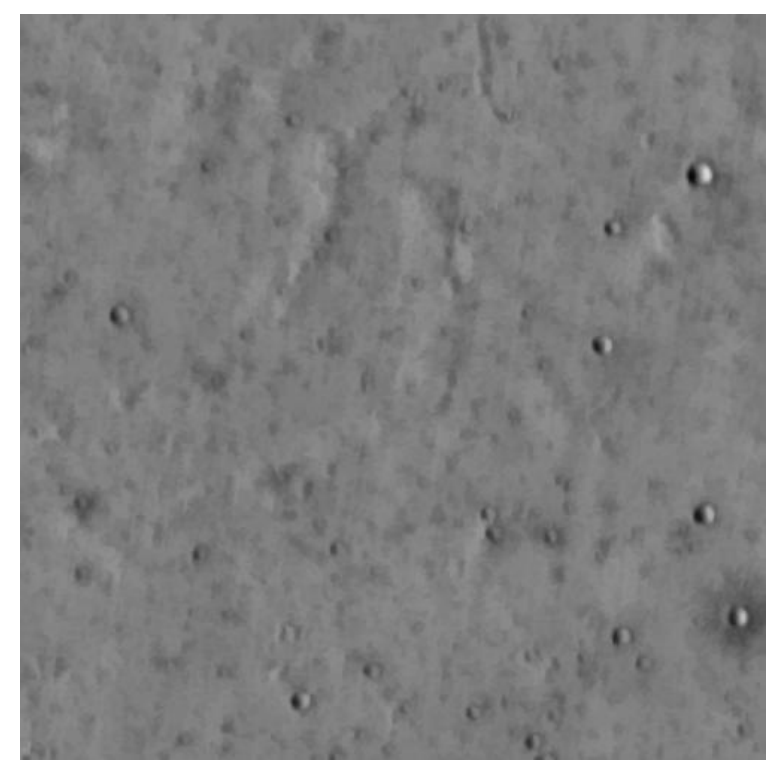

(a)

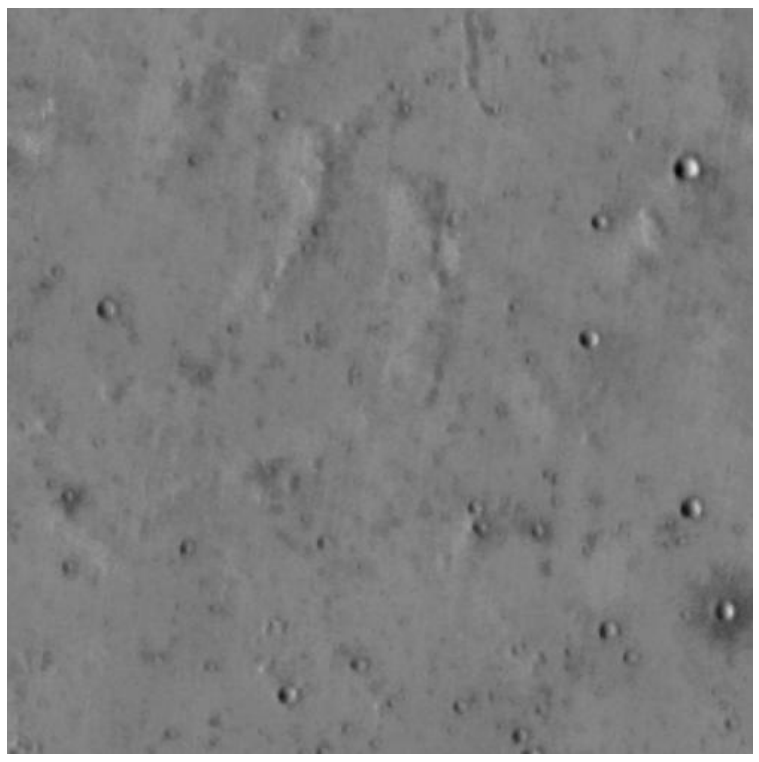

(b)

Figura 1: Resultado da aplicação do filtro bilateral (a) e dos operadores morfológicos: volume máximo e volume mínimo(b)

\subsection{Filtragem}

Para filtrar os alvos que não são de interesse desse trabalho, aplicou-se três operadores morfológicos, fechamento por área, abertura e fechamento.

$\mathrm{O}$ operador fechamento por área tem a finalidade de conectar área menor do que um limiar estipulado. Desta forma, pequenas estruturas foram eliminadas da imagem.

Em seguida, aplicou-se o operador de abertura, o qual consiste da dilatação seguida da erosão, com elemento estruturante disco de raio 2. Assim, grande parte das estruturas que não possuíam a forma circular foi eliminada.

Com o objetivo de recuperar a forma circular das crateras, foi aplicado o operador de fechamento, que consiste da dilatação seguida da erosão. Desta forma, a borda de algumas crateras foi conectada e depois com o processo de erosão, elas recuperaram o seu tamanho original.

$\mathrm{O}$ resultado dessa fase de filtragem é mostrado na Figura 2.

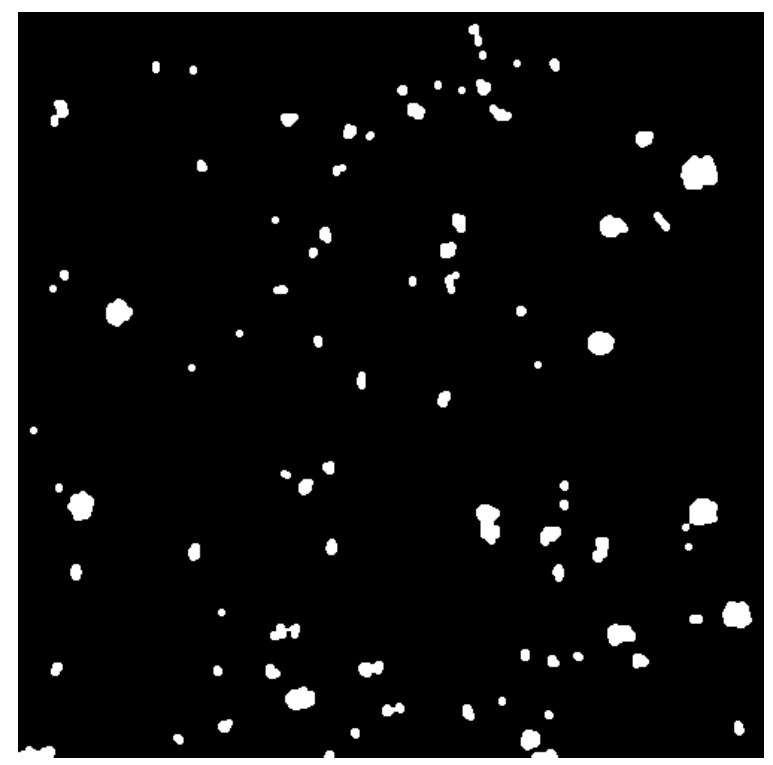

Figura 2: Resultado da etapa de filtragem morfológica.

\subsection{Segmentação}

Esta última fase consistiu em separar alvos que se encontravam próximos e devido à aplicação dos operadores anteriores, foram detectados como um único alvo.

A ferramenta utilizada foi a transformação watershed a partir de marcadores. Os marcadores foram determinados pela aplicação da transformação distancia, a qual cria uma imagem em níveis de cinza, onde cada valor de nível de cinza representa a distancia de determinado pixel com relação ao pixel do fundo mais próximo. Desta forma, os pixels centrais das crateras foram os que tiveram os valores mais 
altos. Assim, aplicou-se o operador região máxima, o qual identificou esses pixels centrais. Cada conjunto de pixels identificados como região máxima foram tomados como marcadores. Na sequencia, a imagem resultante da transformação distancia, foi negativada, o que implicou que os valores mais altos tornaram-se os mínimos, isso por que a transformação watershed consiste da seguinte analogia, na posição de cada marcador é como se houvesse feito um furo na imagem, e a água entra por esses orifícios e preenche a região até que quando águas provenientes de duas regiões distintas se encontram são formadas linhas, essas linhas são denominadas linhas de watershed, Figura 3 (a). A Figura 3 (b) exibe o resultado dessa fase de segmentação.

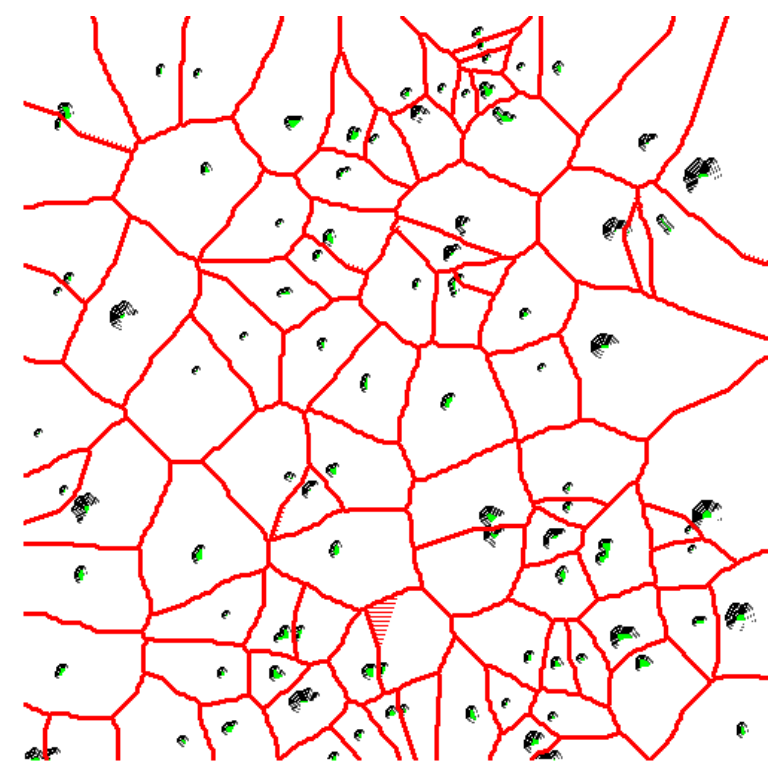

(a)

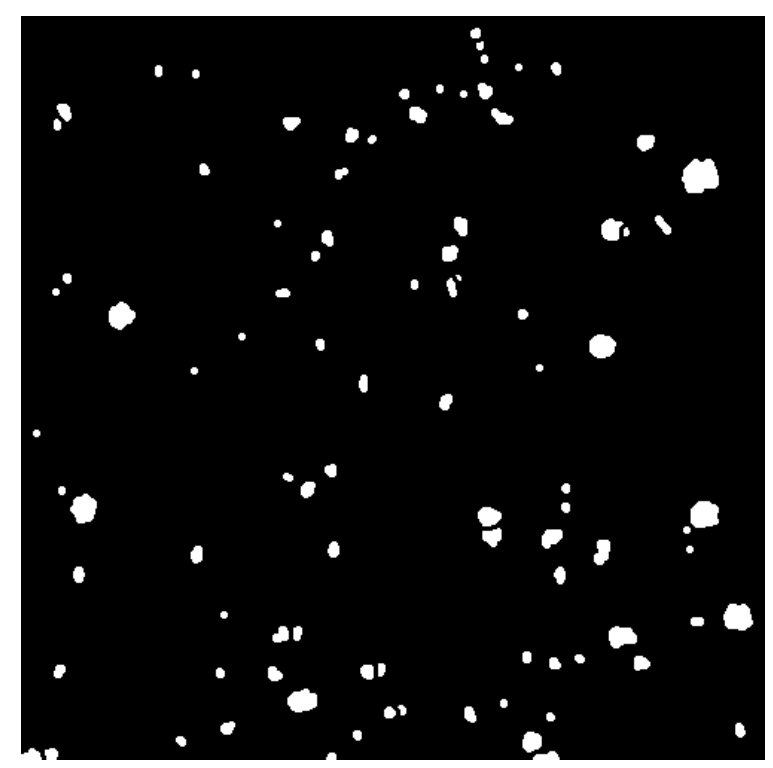

(b)

Figura 3: Resultado da etapa de segmentação por watershed.
$\mathrm{Na}$ Figura 3 (a) os pixels verdes representam as regiões máximas, e as linhas vermelhas representam as linhas de watershed.

Com objetivo de comparar o resultado da rotina combinando o filtro bilateral e operadores morfológicos, com a rotina apenas com operadores morfológicos, os dois resultados foram sobrepostos na imagem original para uma análise visual, conforme apresenta a Figura 4.

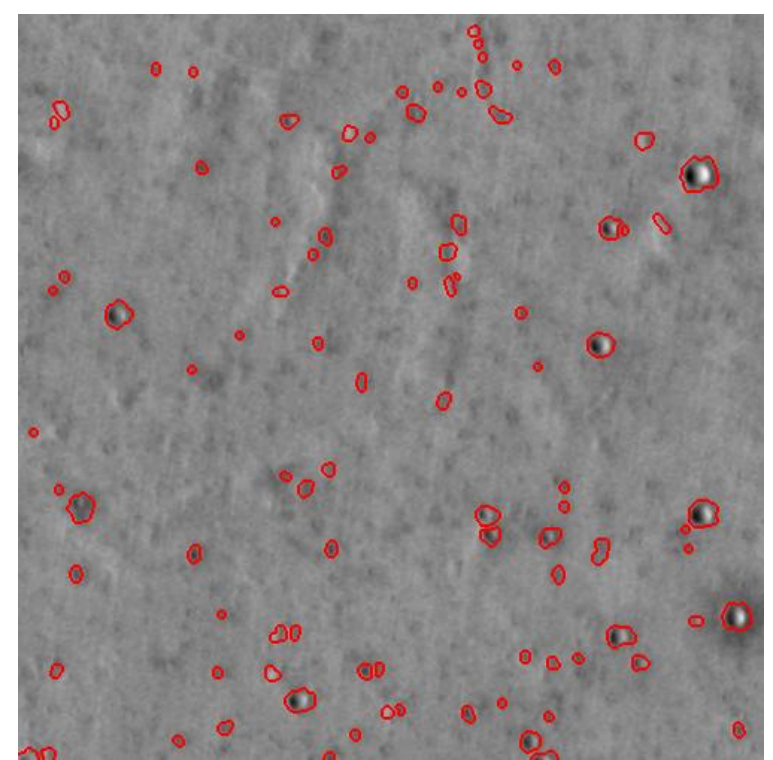

(a)

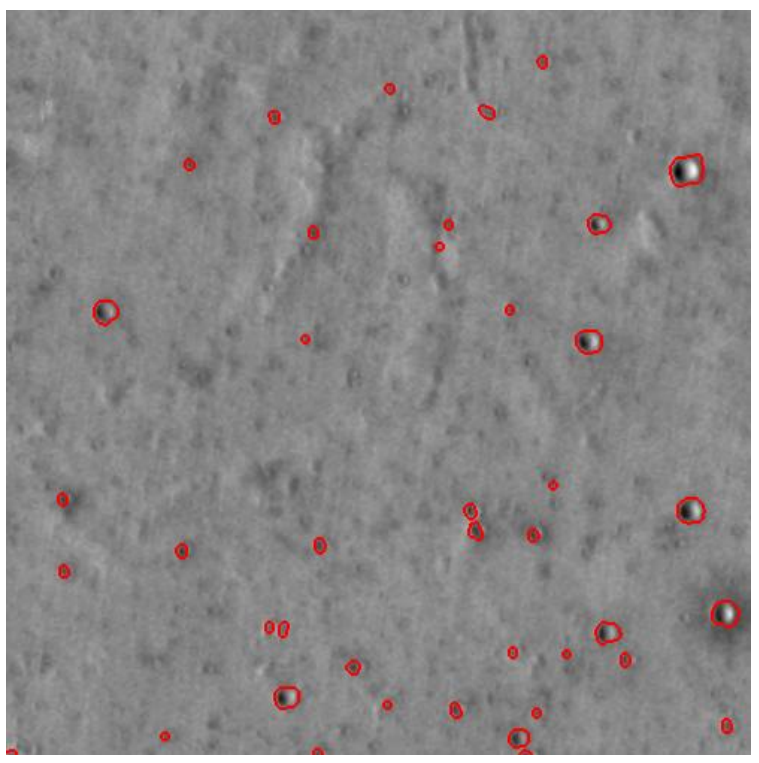

(b)

Figura 4: Resultado final. (a) Rotina combinado filtro bilateral e operadores morfológicos, (b) Rotina utilizando apenas operadores morfológicos. 


\section{Conclusão}

O método desenvolvido é capaz de detectar crateras de impacto em imagens digitais. Para desenvolvimento deste método, vários filtros foram testados e os que apresentaram melhores resultados foi o filtro morfológico e o filtro bilateral. Desta forma, esses dois foram testados em toda a metodologia para verificar qual apresentaria melhor resultado no processo de detecção final. Como pode ser obervado na Figura 4, por meio da análise visual, o método combinando o filtro bilateral com a morfologia matemática, Figura 4 (a) foi mais eficiente do que o que utiliza apenas a morfologia matemática, Figura 4 (b). É possível notar que com a combinação das duas ferramentas, um número maior de crateras de impacto foi detectado. Isso pode ser justificado, pelo fato do filtro bilateral suavizar a imagem preservando a bordas dos alvos, o que possibilitou a detecção de crateras menores. Sendo que no caso da utilização dos filtros morfológicos estas crateras menores foram eliminadas, devido esses filtros não possuir a característica de preservar as bordas dos alvos.

O processo de detecção de crateras possui um elevado grau de complexidade, visto que as crateras possui uma variedade de aspecto, e numa mesma cena existem outros alvos além delas. As crateras sobrepostas e crateras próximas também contribuem para elevar o grau de dificuldade do processo de detecção. Neste sentido, o filtro bilateral se mostrou muito eficiente, pois como ele preserva as bordas, os alvos não são considerados como únicos nas etapas posteriores.

O presente trabalho teve como enfoque principal testar filtros na etapa de pré-processamento para verificar qual contribuiria no processo de detecção, visando alcançar maior quantidade de detecção corretas e menor número de falsas detecções. Esta é uma etapa de um trabalho que está em desenvolvimento. Para as próximas etapas pretende-se testar o método em um maior número de imagens, além de testar outras técnicas de filtragem.

\section{Referências Bibliográficas}

CRÓSTA, A. P. Crateras meteoríticas no Brasil. Textos de Glossário Geológico Ilustrado. Disponível em <http://www.unb.br/ig/glossario/>. Acesso em 01 de agosto de 2012.

FACON, J. Morfologia Matemática: Teoria e Exemplos. Curitiba: PUC,1996.

LOTUFO, R. A.; DOUGHERTY, E. R. Hands on Morphological Image Processing. SPIE Press,. Bellingham, Washington, 2003.

SOILLE, P. Morphological Image Analysis. Berlin. Springer - Verlag, second edition, 2003.

STEPINSKI, T. F., VILALTA, W. D. R. Machine Images Learning Approaches to Detecting Impact Craters in Planetary. Intelligent Data Analy- sis for Real-Life Applications: theory and practice, IGI Global, 2012.

ZHANG, M. Bilateral Filter in Image Processing.2009. Tese (Doutorado em Ciências em Engenharia Elétrica). Faculty of the Louisiana State University and Agricultural and Mechanical College, Louisiana. 